\title{
DIFFUSIVE SHOCK ACCELERATION AT COSMOLOGICAL SHOCK WAVES
}

\author{
Hyesung KAng ${ }^{1}$ AND Dongsu RyU ${ }^{2}$ \\ ${ }^{1}$ Department of Earth Sciences, Pusan National University, Pusan 609-735, Korea; kang@uju.es.pusan.ac.kr \\ 2 Department of Astronomy and Space Science, Chungnam National University, Daejeon 305-764, Korea; ryu@canopus.cnu.ac.kr \\ Received 2012 November 5; accepted 2012 December 13; published 2013 January 29
}

\begin{abstract}
We reexamine nonlinear diffusive shock acceleration (DSA) at cosmological shocks in the large-scale structure of the universe, incorporating wave-particle interactions that are expected to operate in collisionless shocks. Adopting simple phenomenological models for magnetic field amplification (MFA) by cosmic-ray (CR) streaming instabilities and Alfvénic drift, we perform kinetic DSA simulations for a wide range of sonic and Alfvénic Mach numbers and evaluate the CR injection fraction and acceleration efficiency. In our DSA model, the CR acceleration efficiency is determined mainly by the sonic Mach number $M_{s}$, while the MFA factor depends on the Alfvénic Mach number and the degree of shock modification by CRs. We show that at strong CR modified shocks, if scattering centers drift with an effective Alfvén speed in the amplified magnetic field, the CR energy spectrum is steepened and the acceleration efficiency is reduced significantly, compared to the cases without such effects. As a result, the postshock CR pressure saturates roughly at $\sim 20 \%$ of the shock ram pressure for strong shocks with $M_{s} \gtrsim 10$. In the test-particle regime $\left(M_{s} \lesssim 3\right)$, it is expected that the magnetic field is not amplified and the Alfvénic drift effects are insignificant, although relevant plasma physical processes at low Mach number shocks remain largely uncertain.
\end{abstract}

Key words: acceleration of particles - cosmic rays - galaxies: clusters: general - shock waves

Online-only material: color figures

\section{INTRODUCTION}

It is expected that hierarchical gravitational clustering of matter induces shock waves in baryonic gas in the large-scale structure (LSS) of the universe (Kang et al. 1996; Miniati et al. 2000). Simulations for the LSS formation suggest that strong shocks $\left(M_{s} \gtrsim 10\right)$ form in relatively cooler environments in voids, filaments, and outside cluster virial radii, while weak shocks $\left(M_{s} \lesssim\right.$ several) are produced by mergers and flow motions in hotter intracluster media (ICMs; Ryu et al. 2003; Pfrommer et al. 2006; Kang et al. 2007; Skillman et al. 2008; Hoeft et al. 2008; Vazza et al. 2009; Brüggen et al. 2012). Observationally, the existence of such weak shocks in ICMs has been revealed through temperature jumps in the X-ray-emitting gas and $\mathrm{Mpc}$-scale relics with radio spectra softening downstream of the shock (see Markevitch \& Vikhlinin 2007; Feretti et al. 2012, for reviews). These cosmological shocks are the primary means through which the gravitational energy released during the LSS formation is dissipated into the gas entropy, magnetic field, turbulence, and nonthermal particles (Ryu et al. 2008).

In fact, shocks are ubiquitous in astrophysical environments from the heliosphere to galaxy clusters, and they are thought to be the main "cosmic accelerators" of high-energy cosmic-ray (CR) particles (Blandford \& Eichler 1987). In diffusive shock acceleration (DSA) theory, suprathermal particles are scattered by magnetohydrodynamic (MHD) waves and isotropized in the local wave frames and gain energy through multiple crossings of the shock (Bell 1978; Drury 1983; Malkov \& Drury 2001). While most postshock thermal particles are advected downstream, some suprathermal particles energetic enough to swim against downstream turbulent waves can cross the shock and be injected into the Fermi first-order process. Then these streaming CRs generate resonant waves via two-stream instability and nonresonant waves via CR current-driven instability, which in turn amplify turbulent magnetic fields in the preshock region
(Bell 1978, 2004; Lucek \& Bell 2000; Schure et al. 2012). Thin $\mathrm{X}$-ray synchrotron emitting rims observed in several young supernova remnants (SNRs) indicate that CR electrons are accelerated to $10-100 \mathrm{TeV}$ and cool radiatively in the magnetic field of several $100 \mu \mathrm{G}$ behind the forward shock (e.g., Parizot et al. 2006; Reynolds et al. 2012). This provides clear evidence for efficient magnetic field amplification (MFA) during CR acceleration at strong CR modified shocks.

These plasma physical processes, i.e., injection of suprathermal particles into the CR population, excitation of MHD waves and amplification of turbulent magnetic fields via plasma instabilities, and further acceleration of CRs via Fermi first-order process, are important ingredients of DSA and should operate at all types of astrophysical shocks including cosmological shocks in the LSS (e.g., Malkov \& Drury 2001; Zweibel \& Everett 2010; Schure et al. 2012; Brüggen et al. 2012). In addition, relativistic particles can be accelerated stochastically by MHD turbulence, most likely driven in ICMs of merging clusters (Petrosian 2001; Cassano \& Brunetti 2005; Brunetti \& Lazarian 2007). CRs can also be injected into the intergalactic space by radio galaxies (Kronberg et al. 2001) and through winds from star-forming galaxies (Völk \& Atoyan 1999) and later re-accelerated by turbulence and/or shocks. Diffuse synchrotron emission from radio halos and relics in galaxy clusters indicates the presence of $\mathrm{GeV}$ electrons gyrating in $\mu \mathrm{G}$-level magnetic fields on Mpc scales (e.g., Carilli \& Taylor 2002; Govoni \& Feretti 2004; van Weeren et al. 2010; Kang et al. 2012). On the other hand, non-detection of $\gamma$-ray emission from galaxy clusters by Fermi-LAT and VERITAS observations, combined with radio halo observations, puts rather strong constraints on the $\mathrm{CR}$ proton population and the magnetic field strength in ICMs, if one adopts the "hadronic" model, in which inelastic collisions of CR protons with ICM protons produce the radio-emitting electrons and the $\pi^{0}$ decay (Ackermann et al. 2010; Donnert et al. 2010; Jeltema \& Profumo 2011; Arlen et al. 2012). Alternatively, in the "re-acceleration" 
model, in which those secondary electrons produced by $p-p$ collisions are accelerated further by MHD turbulence in ICMs, the CR proton pressure not exceeding a few percent of the gas thermal pressure could be consistent with both the Fermi-LAT upper limits from the $\mathrm{GeV} \gamma$-ray flux and the radio properties of cluster halos (Brunetti et al. 2012).

Recently, amplification of turbulent magnetic fields via plasma instabilities and injection of CR protons and electrons at non-relativistic collisonless shocks have been studied, using particle-in-cell (PIC) and hybrid plasma simulations (e.g., Riquelme \& Spitkovsky 2009, 2011; Guo et al. 2010; Garaté \& Spitkovsky 2012). In PIC simulations, the Maxwell equations for electric and magnetic fields are solved along with the equations of motion for ions and electrons, so the full wave-particle interactions can be followed from first principles. However, extremely wide ranges of length- and timescales need to be resolved mainly because of the large proton-to-electron mass ratio. In hybrid simulations, only the ions are treated kinetically while the electrons are treated as a neutralizing, massless fluid, alleviating severe computational requirements. However, it is still prohibitively expensive to simulate the full extent of DSA from the thermal energies of background plasma to the relativistic energies of $\mathrm{CRs}$, following the relevant plasma interactions at the same time. So we do not yet have full understandings of injection and diffusive scattering of CRs and MFA to make precise quantitative predictions for DSA. Instead, most kinetic DSA approaches, in which the diffusion-convection equation for the phase-space distribution of particles is solved, commonly adopt phenomenological models that may emulate some of those processes (e.g., Kang et al. 2002; Berezhko et al. 2009; Ptuskin et al. 2010; Lee et al. 2012; Caprioli 2012; Kang 2012). Another approximate method is a steady-state Monte Carlo simulation approach, in which parameterized models for particle diffusion, growth of self-generated MHD turbulence, wave dissipation, and plasma heating are implemented (e.g., Vladimirov et al. 2008).

In our previous studies, we performed DSA simulations of $\mathrm{CR}$ protons at cosmological shocks, assuming that the magnetic field strength is uniform in space and constant in time, and presented the time-asymptotic values of fractional thermalization, $\delta\left(M_{s}\right)$, and fractional $\mathrm{CR}$ acceleration, $\eta\left(M_{s}\right)$, as a function of the sonic Mach number $M_{s}$ (Kang \& Jones 2007; Kang et al. 2009). These energy dissipation efficiencies were adopted in a post-processing step for structure formation simulations in order to estimate the CR generation at cosmological shocks (e.g., Skillman et al. 2008; Vazza et al. 2009). Recently, Vazza et al. (2012) have used those efficiencies to include self-consistently the CR pressure terms in the gas-dynamic conservation equations for cosmological simulations. In this paper, we revisit the problem of DSA efficiency at cosmological shocks, including phenomenological models for MFA and drift of scattering centers with Alfvén speed in the amplified magnetic field. Amplification of turbulent magnetic fields driven by CR streaming instabilities is included through an approximate, analytic model suggested by Caprioli (2012). As in our previous works, a thermal leakage injection model and a Bohm-like diffusion coefficient $(\kappa(p) \propto p)$ are adopted as well.

This paper is organized as follows. The numerical method and phenomenological models for plasma physical processes in DSA theory and the model parameters for cosmological shocks are described in Section 2. We then present the detailed simulation results in Section 3 and summarize the main conclusion in Section 4.

\section{DSA MODEL}

In the diffusion approximation, where the pitch-angle distribution of CRs is nearly isotropic, the Fokker-Plank equation of the particle distribution function is reduced to the following diffusion-convection equation:

$\frac{\partial f}{\partial t}+\left(u+u_{w}\right) \frac{\partial f}{\partial x}=\frac{p}{3} \frac{\partial\left(u+u_{w}\right)}{\partial x} \frac{\partial f}{\partial p}+\frac{\partial}{\partial x}\left[\kappa(x, p) \frac{\partial f}{\partial x}\right]$,

where $f(x, p, t)$ is the isotropic part of the pitch-angle-averaged CR distribution function, $\kappa(x, p)$ is the spatial diffusion coefficient along the direction parallel to the mean magnetic field, and $u_{w}$ is the drift speed of local Alfvénic wave turbulence with respect to the plasma (Skilling 1975). Here, we consider quasi-parallel shocks in one-dimensional planar geometry, in which the mean magnetic field is roughly parallel to the flow direction. The flow velocity, $u$, is calculated by solving the momentum conservation equation with dynamical feedback of the CR pressure and self-generated magnetic fields

$$
\frac{\partial(\rho u)}{\partial t}+\frac{\partial\left(\rho u^{2}+P_{g}+P_{c}+P_{B}\right)}{\partial x}=0 .
$$

The CR pressure, $P_{c}$, is calculated self-consistently with the CR distribution function $f$, while the magnetic pressure, $P_{B}$, is calculated according to our phenomenological model for MFA (see Section 2.4) rather than solving the induction equation (Caprioli et al. 2009). We point out that the dynamical effects of magnetic field are not important with $P_{B} \lesssim 0.01 \rho_{0} u_{s}^{2}$. The details of our DSA numerical code, the CRASH (Cosmic-Ray Amr SHock), can be found in Kang et al. (2002).

\subsection{Thermal Leakage Injection}

Injection of protons from the postshock thermal pool into the $\mathrm{CR}$ population via wave-particle interactions is expected to depend on several properties of the shock, including the sonic and Alfvénic Mach numbers, the obliquity angle of mean magnetic field, and the strength of pre-existing and self-excited MHD turbulence. As in our previous studies, we adopt a simple phenomenological model in which particles above an "effective" injection momentum $p_{\text {inj }}$ get injected to the CR population:

$$
p_{\text {inj }} \approx 1.17 m_{p} u_{2}\left(1+\frac{1.07}{\epsilon_{B}}\right),
$$

where $\epsilon_{B}=B_{0} / B_{\perp}$ is the ratio of the mean magnetic field along the shock normal, $B_{0}$, to the amplitude of the postshock MHD wave turbulence, $B_{\perp}$ (Malkov \& Drury 2001; Kang et al. 2002). This injection model reflects plasma physical arguments that the particle speed must be several times larger than the downstream flow speed, $u_{2}$, depending on the strength of MHD wave turbulence, in order for suprathermal particles to leak upstream across the shock transition layer. Since the physical range of the parameter $\epsilon_{B}$ is not tightly constrained, we adopt $\epsilon_{B}=0.25$ as a canonical value, which results in the injected particle fraction $\xi=n_{\mathrm{cr}, 2} / n_{2} \sim 10^{-4}-10^{-3}$ for $M_{s} \gtrsim 3$ (see Figure 3). Previous studies showed that DSA saturates for $\xi \gtrsim 10^{-4}$, so the acceleration efficiency obtained here may represent an upper limit for the efficient injection regime (e.g., Kang et al. 2002; Caprioli 2012). In fact, this injection fraction is similar to the commonly adopted values for nonlinear DSA modeling of SNRs (e.g., Berezhko et al. 2009). If we adopt a smaller value of $\epsilon_{B}$ for stronger wave turbulence, $p_{\text {inj }}$ has to be higher, leading to a smaller injection fraction and a lower acceleration efficiency. 


\subsection{Bohm-like Diffusion Model}

In our model, turbulent MHD waves are self-generated efficiently by plasma instabilities driven by CRs streaming upstream in the shock precursor, so we can assume that CR particles are resonantly scattered by Alfvén waves with fully saturated spectra. Then the particle diffusion can be approximated by a Bohm-like diffusion coefficient, $\kappa_{B} \sim(1 / 3) r_{g} v$, but with flattened non-relativistic momentum dependence (Kang \& Jones 2007):

$$
\kappa(x, p)=\kappa^{*} \frac{B_{0}}{B_{\|}(x)} \cdot \frac{p}{m_{p} c},
$$

where $\kappa^{*}=m_{p} c^{3} /\left(3 e B_{0}\right)=\left(3.13 \times 10^{22} \mathrm{~cm}^{2} \mathrm{~s}^{-1}\right) B_{0}^{-1}$ and $B_{0}$ is the magnetic field strength far upstream expressed in units of $\mu \mathrm{G}$. The strength of the parallel component of the local magnetic field, $B_{\|}(x)$, will be described in the next section. Hereafter, we use the subscripts " 0 ," " 1, " and " 2 " to denote conditions far upstream of the shock, immediate upstream, and downstream of the subshock, respectively.

\subsection{Magnetic Field Amplification}

It was well known that CRs streaming upstream in the shock precursor excite resonant Alfvén waves with a wavelength $(\lambda)$ comparable with the $\mathrm{CR}$ gyroradius $\left(r_{g}\right)$, and turbulent magnetic fields can be amplified into the nonlinear regime (i.e., $\delta B \gg B_{0}$ ) (Bell 1978; Lucek \& Bell 2000). Later, it was discovered that the nonresonant $\left(\lambda \ll r_{g}\right)$, fast-growing instability driven by the CR current $\left(j_{\mathrm{cr}}=e n_{\mathrm{cr}} u_{s}\right)$ can amplify the magnetic field by orders of magnitude, up to the level consistent with the thin X-ray rims at SNRs (Bell 2004). Several plasma simulations have shown that both $B_{\|} / B_{0}$ and $B_{\perp} / B_{0}$ can increase by a factor of up to $\sim 10-45$ via the Bell's CR current-driven instability (Riquelme \& Spitkovsky 2009, 2010; Ohira et al. 2009). Moreover, it was suggested that long-wavelength magnetic fluctuations can grow as well in the presence of short-scale, circularly polarized Alfvén waves excited by the Bell-type instability (Bykov et al. 2011). Recently, Rogachevskii et al. (2012) have also shown that large-scale magnetic fluctuations can grow along the original field by the $\alpha$ effect driven by the nonresonant instability and both the parallel and perpendicular components can be further amplified. There are several other instabilities that may amplify the turbulent magnetic field on scales greater than the CR gyroradius such as the firehose, filamentation, and acoustic instabilities (e.g., Beresnyak et al. 2009; Drury \& Downes 2012; Schure et al. 2012). Although Bell's (2004) original study assumed parallel background magnetic field, it turns out that the non-resonant instability operates for all shocks, regardless of the inclination angle between the shock normal and the mean background magnetic field (Schure et al. 2012), and so the isotropization of the amplified magnetic field can be a reasonable approximation (Riquelme \& Spitkovsky 2009; Rogachevskii et al. 2012).

Here we adopt the prescription for MFA due to CR streaming instabilities that was suggested by Caprioli (2012), based on the assumption of isotropization of the amplified magnetic field and the effective Alfven speed in the local, amplified field: $\delta B^{2} /\left(8 \pi \rho_{0} u_{s}^{2}\right)=(2 / 25)\left(1-U^{5 / 4}\right)^{2} U^{-1.5}$, where $\delta \mathbf{B}=\mathbf{B}-\mathbf{B}_{\mathbf{0}}$ and $U=\left(u_{s}-u\right) / u_{s}$ is the flow speed in the shock rest frame normalized by the shock speed $u_{s}$. In the test-particle regime where the flow structure is not modified, the upstream magnetic field is not amplified in this model (i.e., $U(x)=1$ ). In the shock precursor $\left(x>x_{s}\right.$, where $x_{s}$ is the shock position), the MFA factor becomes

$$
\frac{\delta B(x)^{2}}{B_{0}^{2}}=\frac{4}{25} M_{A, 0}^{2} \frac{\left(1-U(x)^{5 / 4}\right)^{2}}{U(x)^{3 / 2}},
$$

where $M_{A, 0}=u_{s} / v_{A, 0}$ is the Alfvénic Mach number for the far upstream Alfvén speed and $v_{A, 0}=B_{0} / \sqrt{4 \pi \rho_{0}}$. This model predicts that MFA increases with $M_{A, 0}$ and the precursor strength (i.e., degree of shock modification by CRs). In the case of a "moderately modified" shock, in which the immediate preshock speed is $U_{1} \approx 0.8$, for example, the amplified magnetic pressure increases to $\delta B_{1}^{2} / 8 \pi \approx 6.6 \times 10^{-3} \rho_{0} u_{s}^{2}$ and the amplification factor scales as $\delta B_{1} / B_{0} \approx 0.12 M_{A, 0}$. We will show in the next section that the shock structure is modified only moderately owing to the Alfvénic drift, so the magnetic field pressure is less than a few percent of the shock ram pressure even at strong shocks $\left(M_{s} \gtrsim 10\right)$.

For the highest Mach number model considered here, $M_{s}=$ 100 , the preshock amplification factor becomes $\delta B_{1} / B_{0} \approx$ 100 , which is somewhat larger than what was found in the plasma simulations for the Bell-type current-driven instability (Riquelme \& Spitkovsky 2009, 2010). Considering possible MFA beyond the Bell-type instability by other large-scale instabilities (e.g., Bykov et al. 2011; Rogachevskii et al. 2012; Schure et al. 2012), this level of MFA may not be out of reach. Note that this recipe is intended to be a heuristic model that may represent qualitatively the MFA process in the shock precursor.

Assuming that the two perpendicular components of preshock magnetic fields are completely isotropized and simply compressed across the subshock, the immediate postshock field strength can be estimated by

$$
B_{2} / B_{1}=\sqrt{1 / 3+2 / 3\left(\rho_{2} / \rho_{1}\right)^{2}} .
$$

We note that the MFA model described in Equations (5) and (6) is also used for the diffusion coefficient model given by Equation (4).

\subsection{Alfvénic Drift}

Resonant Alfvén waves excited by the $\mathrm{CR}$ streaming are pushed by the CR pressure gradient $\left(\partial P_{c} / \partial x\right)$ and propagate against the underlying flow in the shock precursor (e.g., Skilling 1975; Bell 1978). The mean drift speed of scattering centers is commonly approximated as the Alfvén speed, i.e., $u_{w, 1}(x) \approx$ $+v_{A} \approx B(x) / \sqrt{4 \pi \rho(x)}$, pointing upstream away from the shock, where $B(x)$ is the local, amplified magnetic field strength estimated by Equation (5). For isotropic magnetic fields, the parallel component would be roughly $B_{\|} \approx B(x) / \sqrt{3}$. But we simply use $B(x)$ for the effective Alfvén speed, since the uncertainty in this model is probably greater than the factor of $\sqrt{3}$ (see Section 3 for a further comment on this factor). In the postshock region the Alfvénic turbulence is probably relatively balanced, so the wave drift can be ignored, that is, $u_{w, 2} \approx 0$ (Jones 1993). Since the Alfvénic drift reduces the velocity difference between upstream and downstream scattering centers, compared to that of the bulk flow, the resulting CR spectrum becomes softer than estimated without considering the wave drift. Here, we do not consider loss of turbulent magnetic energy and gas heating due to wave dissipation in order to avoid introducing additional free parameters to the problem. 


\subsection{Setup for DSA Simulations}

Previous studies have shown that the DSA efficiency depends primarily on the shock sonic Mach number (Kang et al. 2007). So we considered shocks with a wide range of the sonic Mach number, $M_{s}=1.5-100$, propagating into the intergalactic medium (IGM) of different temperature phases, $T_{0}=10^{4}-5 \times 10^{7} \mathrm{~K}$ (Kang et al. 2005). Then, the shock speed is given by $u_{s}=\left(150 \mathrm{~km} \mathrm{~s}^{-1}\right) M_{s}\left(T_{0} / 10^{6} \mathrm{~K}\right)^{1 / 2}$.

We specify the background magnetic field strength by setting the so-called plasma beta, $\beta_{P}=P_{g} / P_{B}$, the ratio of the gas pressure to the magnetic pressure. So the upstream magnetic field strength is given as $B_{0}^{2}=8 \pi P_{g} / \beta_{P}$, where $\beta_{P} \sim 100$ is taken as a canonical value in ICMs (see, e.g., Ryu et al. 2008). Then, the ratio of the background Alfvén speed to the sound speed, $v_{A, 0} / c_{s}=\sqrt{2 /\left(\beta_{P} \gamma_{g}\right)}$ (where $\gamma_{g}$ is the gas adiabatic index), which determines the significance of Alfvénic drift, depends only on the parameter $\beta_{P}$. Moreover, the upstream Alfvénic Mach number, $M_{A, 0}=u_{s} / v_{A, 0}=M_{s} \sqrt{\beta_{P} \gamma_{g} / 2}$, controls the MFA factor as given in Equation (5). For $\beta_{P}=100$ and $\gamma_{g}=5 / 3$, the background Aflvén speed is about $10 \%$ of the sound speed, i.e., $v_{A, 0}=0.11 c_{s}$ (independent of $M_{s}$ and $T_{0}$ ), and $M_{A, 0}=9.1 M_{s}$. For a higher value of $\beta_{P}$ (i.e., weaker magnetic fields), of course, the Alfvénic drift effect will be less significant.

With a fixed value of $\beta_{P}$, the upstream magnetic field strength can be specified by the upstream gas pressure, $n_{\mathrm{H}, 0} T_{0}$, as follows:

$$
B_{0}=0.28 \mu \mathrm{G}\left(\frac{n_{\mathrm{H}, 0} T_{0}}{10^{3} \mathrm{~cm}^{-3} \mathrm{~K}}\right)^{1 / 2}\left(\frac{100}{\beta_{P}}\right)^{1 / 2}
$$

We choose the hydrogen number density, $n_{\mathrm{H}, 0}=10^{-4} \mathrm{~cm}^{-3}$, as the fiducial value to obtain specific values of magnetic field strength shown in Figures 1 and 2. But this choice does not affect the time-asymptotic results shown in Figures 3 and 4, since the CR modified shock evolves in a self-similar manner and the time-asymptotic states depend primarily on $M_{s}$ and $M_{A, 0}$, independent of the specific value of $B_{0}$.

Since the tension in the magnetic field lines hinders Bell's CR current-driven instability, MFA occurs if the background field strength satisfies the condition $B_{0}<B_{s}=(0.87 \mu \mathrm{G})\left(n_{\mathrm{cr}} u_{s}\right)^{1 / 2}$ (Zweibel \& Everett 2010). For a typical shock speed of $u_{s} \sim$ $10^{3} \mathrm{~km} \mathrm{~s}^{-1}$ formed in the IGM with $n_{\mathrm{H}, 0} \sim 10^{-6}-10^{-4} \mathrm{~cm}^{-3}$ with the $\mathrm{CR}$ injection fraction, $\xi \sim 10^{-4}-10^{-3}$, the maximum magnetic field for the growth of nonresonant waves is roughly $B_{s} \sim 0.1-1 \mu \mathrm{G}$. The magnetic field strength estimated by Equation (7) is $B_{0} \approx 0.28 \mu \mathrm{G}$ for $n_{\mathrm{H}, 0}=10^{-4} \mathrm{~cm}^{-3}$ and $T_{0}=10^{7} \mathrm{~K}(\mathrm{ICMs})$ and $B_{0} \approx 10^{-3} \mu \mathrm{G}$ for $n_{\mathrm{H}, 0}=10^{-6} \mathrm{~cm}^{-3}$ and $T_{0}=10^{4} \mathrm{~K}$ (voids). Considering the uncertainties in the model and the parameters, it seems reasonable to assume that MFA via CR streaming instabilities can be effective at cosmological shocks in the LSS (Zweibel \& Everett 2010).

In the simulations, the diffusion coefficient, $\kappa^{*}$ in Equation (4), can be normalized with a specific value of $\kappa_{o}$. Then, the related length- and timescales are given as $l_{o}=\kappa_{o} / u_{s}$ and $t_{o}=\kappa_{o} / u_{s}^{2}$, respectively. Since the flow structure and the CR pressure approach the time-asymptotic self-similar states, a specific physical value of $\kappa_{o}$ matters only in the determination of $p_{\max } / m_{p} c \approx 0.1 u_{s}^{2} t / \kappa^{*}$ at a given simulation time. For example, with $\kappa_{o}=10^{6} \kappa^{*}$, the highest momentum reached at time $t$ becomes $p_{\max } / m_{p} c \approx 10^{5}\left(t / t_{o}\right)$.

It was suggested that nonlinear wave damping and dissipation due to ion-neutral collisions may weaken stochastic scatterings, leading to slower acceleration and escape of highest energy
Table 1

Models

\begin{tabular}{lll}
\hline \hline$T_{0}(\mathrm{~K})$ & \multicolumn{1}{c}{$P_{c, 0}=0$} & $P_{c, 0}=0.05 P_{g, 0}$ \\
\hline $5 \times 10^{7}$ & $M_{s}=1.5,2,3,4$ & $M_{s}=1.5,2,3$ \\
$10^{7}$ & $M_{s}=3,4,5$ & $M_{s}=4,5$ \\
$10^{6}$ & $M_{s}=5,7,10$ & $M_{s}=7,10$ \\
$10^{5}$ & $M_{s}=10,20,30,50$ & $M_{s}=20,30,50$ \\
$10^{4}$ & $M_{s}=20,50,100$ & $M_{s}=100$ \\
\hline
\end{tabular}

particles from the shock (Ptuskin \& Zirakashvili 2005). Since these processes are not well understood in a quantitative way, we do not include wave dissipation in the simulations. Instead, we implement a free escape boundary (FEB) at an upstream location by setting $f\left(x_{\mathrm{FEB}}, p\right)=0$ at $x_{\mathrm{FEB}}=0.5 l_{o}$, which may emulate the escape of the highest energy particles with the diffusion length, $\kappa(p) / u_{s} \gtrsim x_{\mathrm{FEB}}$. Under this FEB condition, the $\mathrm{CR}$ spectrum and the shock structure including the precursor approach the time-asymptotic states in the timescale of $t / t_{o} \sim 1$ (Kang 2012).

As noted in the introduction, CR protons can be accelerated by merger and accretion shocks, injected into the intergalactic space by star-forming galaxies and active galaxies, and accelerated by turbulence. Because of long lifetime and slow diffusion, CR protons should be accumulated in the LSS over cosmological times. So it seems natural to assume that ICMs contain preexisting populations of $\mathrm{CR}$ protons. But their nature is not well constrained, except that the pressure of $\mathrm{CR}$ protons is less than a few percent of the gas thermal pressure (Arlen et al. 2012; Brunetti et al. 2012). For a model spectrum of pre-existing CR protons, we adopt a simple power-law form, $f_{0}(p)=f_{\text {pre }} \cdot\left(p / p_{\text {inj }}\right)^{-s}$ for $p \geqslant p_{\text {inj }}$, with the slope $s=4.5$, which corresponds to the slope of the test-particle power-law momentum spectrum accelerated at $M=3$ shocks. We note that the slope of the $\mathrm{CR}$ proton spectrum inferred from the radio spectral index (i.e., $\left.\alpha_{R} \approx(s-2) / 2\right)$ of cluster halos is in the range $4.5 \lesssim s \lesssim 5$ (e.g., Jeltema \& Profumo 2011). The amplitude, $f_{\text {pre }}$, is set by the ratio of the upstream CR to gas pressure, $R \equiv P_{c, 0} / P_{g, 0}$, where $R=0.05$ is chosen as a canonical value.

Table 1 lists the considered models: the weak shock models with $T_{0} \geqslant 10^{7} \mathrm{~K}$, the strong shock models with $T_{0}=10^{5}-10^{6} \mathrm{~K}$, and the strongest shock models with $T_{0}=10^{4} \mathrm{~K}$ represent shocks formed in hot ICMs, in the warm-hot intergalactic medium of filaments, and in voids, respectively. Simulations start with purely gasdynamic shocks initially at rest at $x_{s}=0$.

\section{DSA SIMULATION RESULTS}

Figures 1 and 2 show the spatial profiles of magnetic field strength, $B(x)$, and CR pressure, $P_{c}(x)$, and the distribution function of CRs at the shock location, $g_{s}(p)$, at $t / t_{o}=0.5,1,2$ for models without or with pre-existing CRs: from top to bottom panels, $M_{s}=3$ and $T_{0}=5 \times 10^{7} \mathrm{~K}, M_{s}=5$ and $T_{0}=10^{7} \mathrm{~K}$, $M_{s}=10$ and $T_{0}=10^{6} \mathrm{~K}$, and $M_{s}=100$ and $T_{0}=10^{4} \mathrm{~K}$. Note that the models with $M_{s}=3-5$ represent shocks formed in hot ICMs, while those with $M_{s}=10$ and 100 reside in filaments and voids, respectively.

The background magnetic field strength corresponds to $B_{0}=$ $0.63,0.28,0.089$, and $8.9 \times 10^{-3} \mu \mathrm{G}$ for the models with $M_{s}=3,5,10$, and 100 , respectively, for the fiducial value of $n_{\mathrm{H}, 0}=10^{-4} \mathrm{~cm}^{-3}$ (see Equation (7)). With our MFA model the postshock field can increase to $B_{2} \approx 2-3 \mu \mathrm{G}$ for all these models, which is similar to the field strengths observed in radio halos and radio relics. The postshock CR pressure increases 

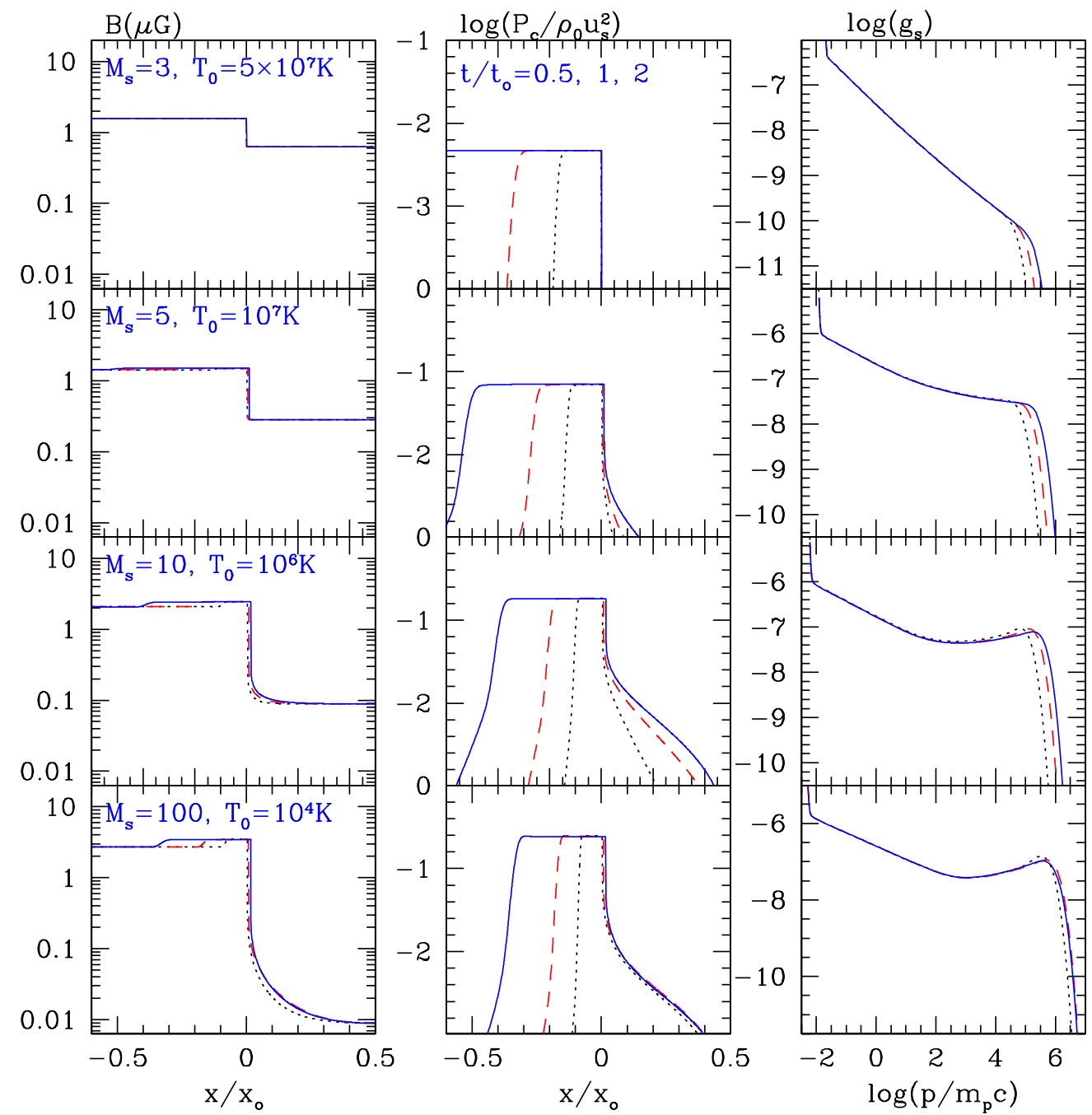

Figure 1. Magnetic field strength, CR pressure profile, and the CR distribution at the shock location at $t / t_{o}=0.5$ (dotted lines), 1 (dashed), and 2 (solid) for models without pre-existing CRs. See Table 1 for the model parameters.

(A color version of this figure is available in the online journal.)

with the sonic Mach number but saturates at $P_{c, 2} /\left(\rho_{0} u_{s}^{2}\right) \approx 0.2$ for $M_{s} \gtrsim 10$. One can see that the precursor profile and $g_{s}(p)$ have reached the time-asymptotic states for $t / t_{o} \gtrsim 1$ for the $M_{s}=100$ model, while the lower Mach number models are still approaching the steady states at $t / t_{o}=2$. This is because in the $M_{s}=100$ model, by the time $t / t_{o} \approx 1$ the CR spectrum has extended to $p_{\max }$ that satisfies the FEB condition. For strong shocks of $M_{s}=10-100$, the power-law index, $q \equiv-\partial \ln f / \partial \ln p$, is about 4.3-4.4 at $p \sim m_{p} c$ instead of $q=4$, because the Alfvénic drift steepens the CR spectrum.

For the models with pre-existing CRs in Figure 2, the pre-existing population is important only for weak shocks with $M_{s} \lesssim 5$, because the injected population dominates in shocks with higher sonic Mach numbers. As mentioned in the introduction, the signatures of shocks observed in ICMs through $\mathrm{X}$-ray and radio observations can be interpreted by low Mach number shocks (Markevitch \& Vikhlinin 2007; Feretti et al. 2012). In particular, the presence of pre-existing CRs is expected to be crucial in explaining the observations of radio relics (Kang et al. 2012).

Figure 3 shows time-asymptotic values of downstream gas pressure, $P_{g, 2}$, and CR pressure, $P_{c, 2}$, in units of $\rho_{0} u_{s}^{2}$, density compression ratios, $\sigma_{1}=\rho_{1} / \rho_{0}$ and $\sigma_{2}=\rho_{2} / \rho_{0}$, the ratios of amplified magnetic field strengths to background strength, $B_{2} / B_{0}$ and $B_{1} / B_{0}$, and postshock CR number fraction, $\xi=$ $n_{\mathrm{cr}, 2} / n_{2}$, as a function of $M_{s}$ for all the models listed in Table 1. We note that for the models without pre-existing CRs (left column) two different values of $T_{0}$ (and so $u_{s}$ ) are considered for each of $M_{s}=3,4,5,10,30$, and 50 models, in order to explore the dependence on $T_{0}$ for a given sonic Mach number. The figure demonstrates that the DSA efficiency and the MFA factor are determined primarily by $M_{s}$ and $M_{A, 0}$, respectively, almost independent of $T_{0}$. For instance, the two Mach 10 models with $T_{0}=10^{5} \mathrm{~K}$ (open triangle) and $10^{6} \mathrm{~K}$ (filled triangle) show similar results as shown in the left column of Figure 3. But note that the curves for $P_{c r, 2}$ and $\xi$ increase somewhat unevenly near $M_{s} \approx$ 4-7 for the models with pre-existing CRs in the right column, because of the change in $T_{0}$ (see Table 1 ).

At weak shocks with $M_{s} \lesssim 3$, the injection fraction is $\xi \lesssim 10^{-4}$ and the CR pressure is $P_{c, 2} / \rho_{0} u_{s}^{2} \lesssim 5 \times 10^{-3}$ without pre-existing CRs, while both values depend on $P_{c, 1}$ in the presence of pre-existing CRs. Since the magnetic field is not amplified in the test-particle regime, these results remain similar to what we reported earlier in Kang \& Ryu (2011). For a larger value of $\epsilon_{B}$, the injection fraction and the CR acceleration efficiency would increase. As shown in Kang \& Jones (2007), 

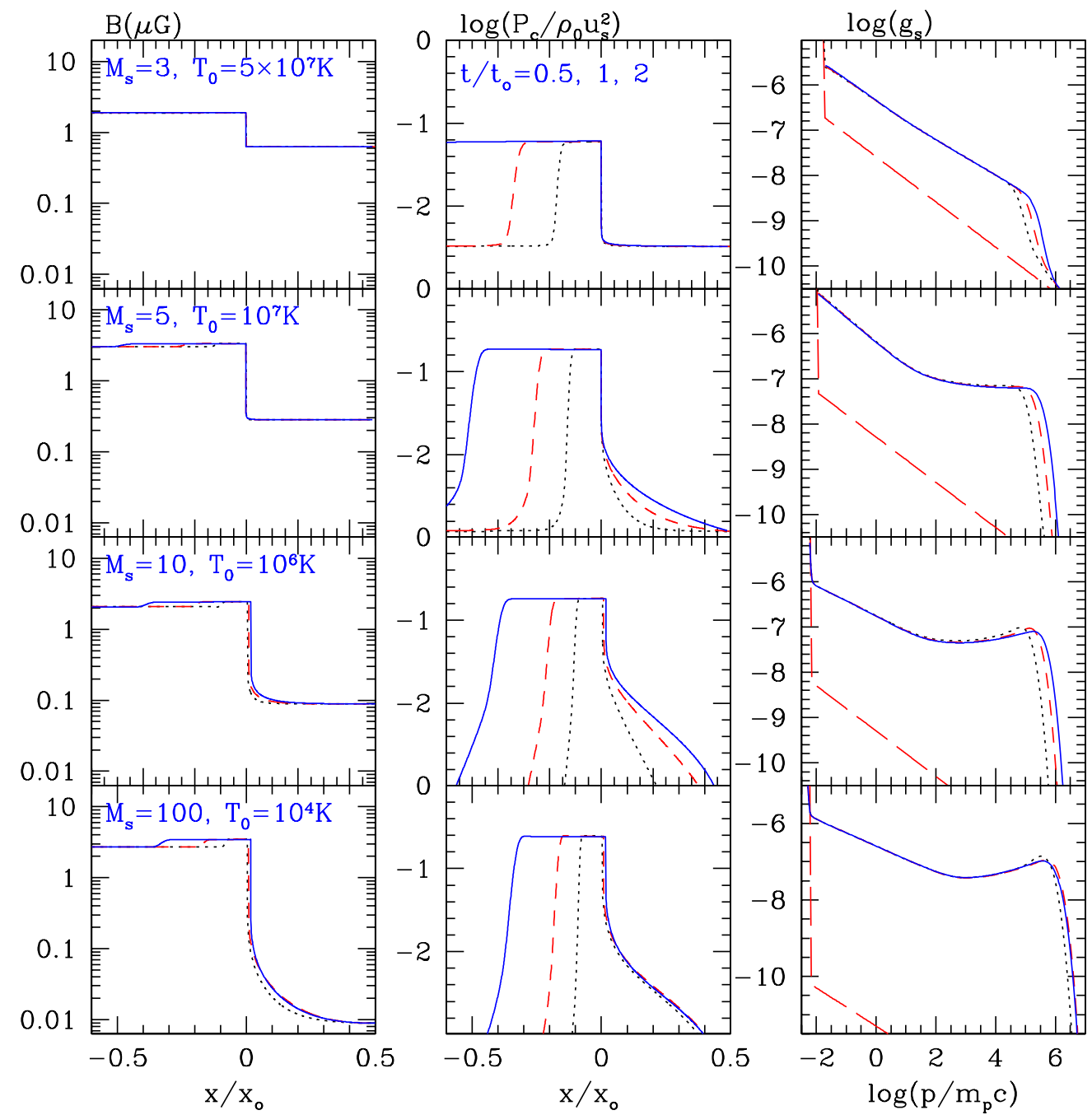

Figure 2. Same as Figure 1 except that models with pre-existing CRs are shown. The pre-existing population has a power-law spectrum, $f_{p} \propto p^{-4.5}$, that corresponds to an upstream CR pressure, $P_{c, 0}=0.05 P_{g, 0}$. In the right column, the long-dashed lines show the pre-existing population.

(A color version of this figure is available in the online journal.)

however, $\xi$ and $P_{c r, 2} / \rho_{0} u_{s}^{2}$ depend sensitively on the injection parameter $\epsilon_{B}$ for $M_{s} \lesssim 5$, while such dependence becomes weak for $M_{s} \gtrsim 10$. Furthermore, there are large uncertainties in the thermal leakage injection model especially at weak shocks. Thus, it is not possible or meaningful to discuss the quantitative dependence of these results on $\epsilon_{B}$, until we obtain more realistic pictures of the wave-particle interactions through PIC or hybrid plasma simulations of weak collisionless shocks.

In the limit of large $M_{s}$, the postshock CR pressure saturates at $P_{c, 2} \approx 0.2 \rho_{0} u_{s}^{2}$, the postshock density compression ratio at $\sigma_{2} \approx 5$, and the postshock CR number fraction at $\xi \approx 2 \times 10^{-3}$. The MFA factors are $B_{1} / B_{0} \sim 0.12 M_{A, 0} \sim M_{s}$ and $B_{2} / B_{0} \sim$ $3 M_{s}$ for $M_{s} \gtrsim 5$, as expected from Equation (5). In Kang et al. (2007) we found that $P_{c, 2} \approx 0.55 \rho_{0} u_{s}^{2}$ in the limit of large $M_{s}$, when the magnetic field strength was assumed to be uniform in space and constant in time. Here, we argue that MFA and Alfvénic drift in the amplified magnetic field steepen the CR spectrum and reduce the DSA efficiency drastically.

Again, the presence of pre-existing CRs (right column) enhances the injection fraction and acceleration efficiency at weak shocks of $M_{s} \lesssim 5$, while it does not affect the results at stronger shocks. Since the upstream $\mathrm{CR}$ pressure is $P_{c, 0}=0.05 P_{g, 0}=\left(0.03 / M_{s}\right) \rho_{0} u_{s}^{2}$ in these models, the enhancement factor $P_{c, 2} / P_{c, 0} \approx 1.5-6$ for $M_{s} \leqslant 3$. So the DSA acceleration efficiency exceeds only slightly the adiabatic compression factor, $\sigma_{2}^{\gamma_{c}}$, where $\gamma_{c} \approx 4 / 3$ is the adiabatic index of the CR population.

As in Kang et al. (2007), the gas thermalization and CR acceleration efficiencies are defined as the ratios of the gas thermal and CR energy fluxes to the shock kinetic energy flux:

$$
\begin{aligned}
\delta\left(M_{s}\right) & \equiv \frac{\left[e_{g, 2}-e_{g, 0}\left(\rho_{2} / \rho_{0}\right)^{\gamma_{s}}\right] u_{2}}{(1 / 2) \rho_{0} u_{s}^{3}}, \\
\eta\left(M_{s}\right) & \equiv \frac{\left[e_{c, 2}-e_{c, 0}\left(\rho_{2} / \rho_{0}\right)^{\gamma_{c}}\right] u_{2}}{(1 / 2) \rho_{0} u_{s}^{3}},
\end{aligned}
$$

where $e_{g}$ and $e_{c}$ are the gas thermal and CR energy densities. The second terms inside the brackets subtract the effect of adiabatic compression that occurred at the shock. Alternatively, the energy dissipation efficiencies not excluding the effect of adiabatic compression across the shock can be defined as

$$
\delta^{\prime}\left(M_{s}\right) \equiv \frac{\left[e_{g, 2} u_{2}-e_{g, 0} u_{0}\right]}{(1 / 2) \rho_{0} u_{s}^{3}}, \quad \eta^{\prime}\left(M_{s}\right) \equiv \frac{\left[e_{c, 2} u_{2}-e_{c, 0} u_{0}\right]}{(1 / 2) \rho_{0} u_{s}^{3}},
$$



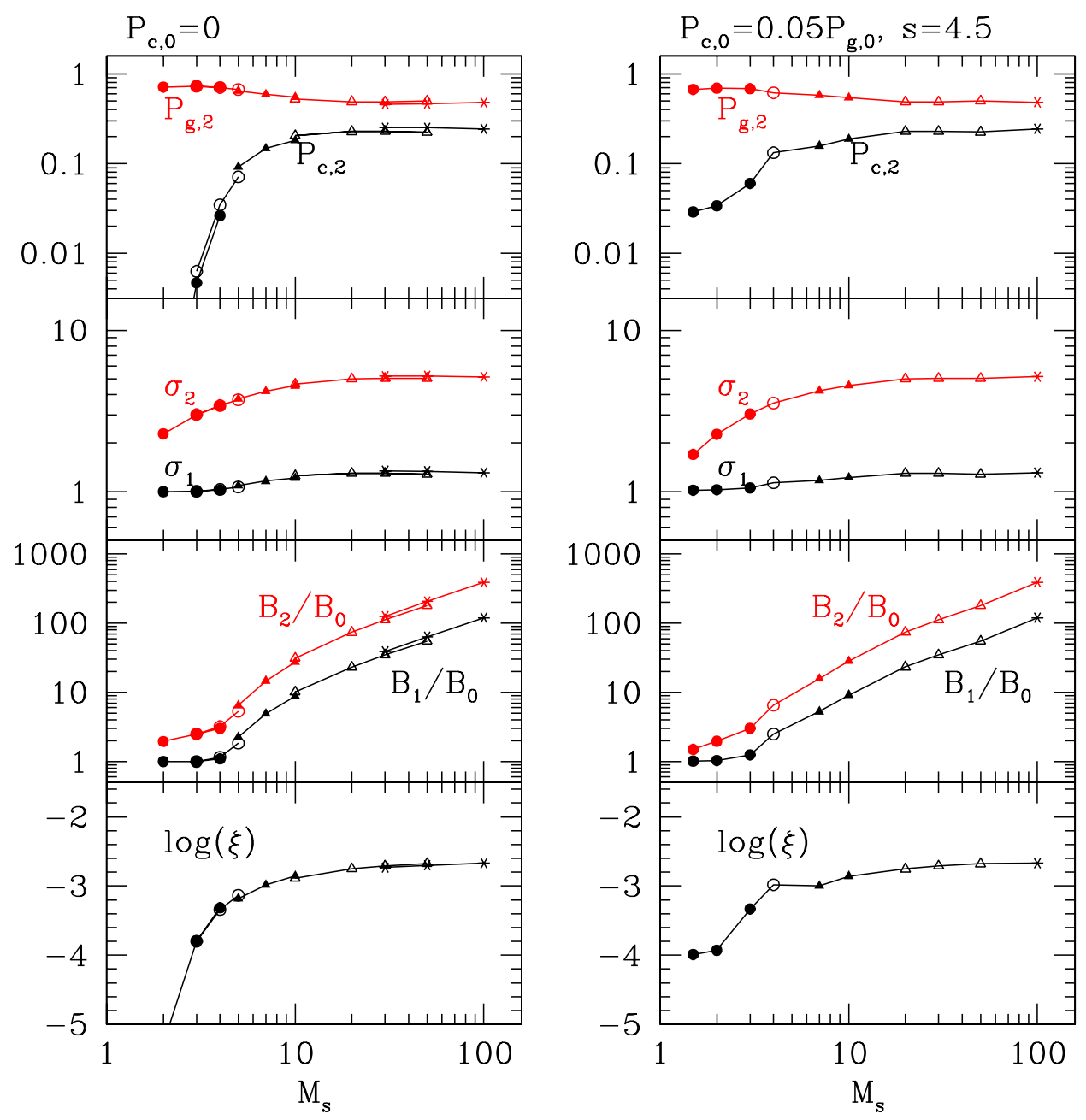

Figure 3. Time-asymptotic values of downstream gas pressure, $P_{g, 2}$, and CR pressure, $P_{c, 2}$, in units of $\rho_{0} u_{s}^{2}$, density compression ratios, $\sigma_{1}=\rho_{1} / \rho_{0}$ and $\sigma_{2}=\rho_{2} / \rho_{0}$, the ratios of amplified magnetic field strengths to background strength, $B_{2} / B_{0}$ and $B_{1} / B_{0}$, and postshock CR number fraction, $\xi$, as a function of the sonic Mach number, $M_{s}$. The left column shows the cases without pre-existing CRs, while the right column shows the cases with pre-existing CRs. Filled circles are used for the models with $T_{0}=5 \times 10^{7} \mathrm{~K}$, open circles for $T_{0}=10^{7} \mathrm{~K}$, filled triangles for $T_{0}=10^{6} \mathrm{~K}$, open triangles for $T_{0}=10^{5} \mathrm{~K}$, and stars for $T_{0}=10^{4} \mathrm{~K}$.

(A color version of this figure is available in the online journal.)
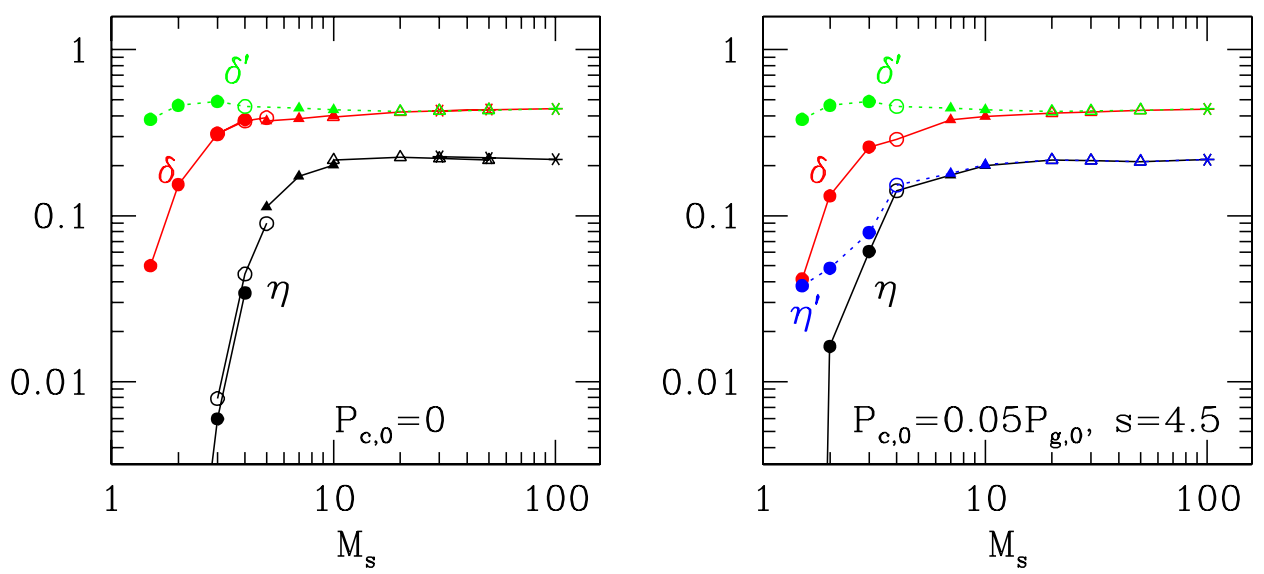

Figure 4. Shock dissipation efficiencies in the form of gas and CR energies, $\delta$ and $\eta$ (solid lines), respectively, in Equation (8) and $\delta^{\prime}$ and $\eta^{\prime}$ (dotted lines) in Equation (9) as a function of the shock sonic Mach number. For the models with different preshock temperature, $T_{0}$, the same symbols are used as in Figure 3 .

(A color version of this figure is available in the online journal.)

which may provide more direct measures of the energy generation at the shock. Note that $\eta=\eta^{\prime}$ for the models with $P_{c, 0}=0$.

Figure 4 shows these dissipation efficiencies for all the models listed in Table 1. Again, the CR acceleration efficiency saturates at $\eta \approx 0.2$ for $M_{s} \gtrsim 10$, which is much lower than what we reported in the previous studies without MFA (Ryu et al. 2003; Kang et al. 2007). The CR acceleration efficiency is $\eta<0.01$ for weak shocks $\left(M_{s} \lesssim 3\right)$ if there are no pre-existing CRs. But the 
efficiency $\eta^{\prime}$ can be as high as 0.1 even for these weak shocks, depending on the amount of pre-existing CRs. The efficiency $\eta$ for weak shocks is not affected by the new models of MFA and Alfvénic drift, since the magnetic field is not amplified in the test-particle regime.

If we choose a smaller value of $\beta_{P}$, the ratio $v_{A, 0} / c_{s}$ is larger, leading to less efficient acceleration due to the stronger Afvénic drift effects. For example, for $\beta_{P} \sim 1$ (i.e., equipartition fields), which is relevant for the interstellar medium in galaxies, the $\mathrm{CR}$ acceleration efficiency in the strong shock limit reduces to $\eta \approx 0.12$ (Kang 2012). On the other hand, if we were to choose a smaller wave drift speed, the CR efficiency $\eta$ will increase slightly. For example, if we choose $u_{w} \approx 0.3 v_{A}$ instead of $u_{w} \approx v_{A}$, the value of $\eta$ in the high Mach number limit would increase to $\sim 0.25$ for the models considered here.

On the other hand, if we choose a smaller injection parameter, for example, $\epsilon_{B}=0.23$, the injection fraction reduces from $\xi=$ $2.1 \times 10^{-4}$ to $6.2 \times 10^{-5}$ and the postshock CR pressure decreases from $P_{c, 2} / \rho_{0} u_{s}^{2}=0.076$ to 0.043 for the $M_{s}=5$ model, while $\xi=2.2 \times 10^{-3}$ to $3.3 \times 10^{-4}$ and $P_{c, 2} / \rho_{0} u_{s}^{2}=0.18$ to 0.14 for the $M_{s}=50$ model. Considering that the $\mathrm{CR}$ injection fraction obtained in these simulations $\left(\xi>10^{-4}\right)$ is in the saturation limit of DSA, the CR acceleration efficiency, $\eta$, for $M \gtrsim 10$ in Figure 4 should be regarded as an upper limit.

\section{SUMMARY}

We revisited the nonlinear DSA of CR protons at cosmological shocks in the LSS, incorporating some phenomenological models for MFA due to CR streaming instabilities and Alfvénic drift in the shock precursor. Our DSA simulation code, CRASH, adopts the Bohm-like diffusion and thermal leakage injection of suprathermal particles into the CR population.

A wide range of preshock temperature, $10^{4} \leqslant T_{0} \leqslant 5 \times 10^{7} \mathrm{~K}$, is considered to represent shocks that form in clusters of galaxies, filaments, and voids. We found that the DSA efficiency is determined mainly by the sonic Mach number $M_{s}$, but almost independent of $T_{0}$. We assumed the background intergalactic magnetic field strength, $B_{0}$, that corresponds to the plasma beta $\beta_{P}=100$. This is translated to the ratio of the Alfvén speed in the background magnetic field to the preshock sound speed, $v_{A, 0} / c_{s}=\sqrt{6 / 5 \beta_{P}} \approx 0.11$. Then the Alfvénic Mach number $M_{A, 0}=\sqrt{5 \beta_{P} / 6} M_{s}$ determines the extent of MFA (i.e., $B_{1} / B_{0}$ ), which in turn controls the significance of Alfvénic drift in DSA. Although the preshock density is set to be $n_{\mathrm{H}, 0}=10^{-4} \mathrm{~cm}^{-3}$ just to give a characteristic scale to the magnetic field strength in the IGM, our results for the CR proton acceleration, such as the dissipation efficiencies, do not depend on a specific choice of $n_{\mathrm{H}, 0}$. If one is interested in CR electrons, which are affected by synchrotron and inverse Compton cooling, the electron energy spectrum should depend on the field strength $B_{0}$ and hence on the value of $n_{\mathrm{H}, 0} T_{0}$ (see Equation (7)).

The main results of this study can be summarized as follows:

1. With our phenomenological models for DSA, the injected fraction of CR particles is $\xi \approx 10^{-4}-10^{-3}$ and the postshock CR pressure becomes $10^{-3} \lesssim P_{c, 2} /\left(\rho_{0} u_{s}^{2}\right) \lesssim 0.2$ for $3 \leqslant M_{s} \leqslant 100$, if there are no pre-existing CRs. A population of pre-existing CRs provides seed particles to the Fermi process, so the injection fraction and acceleration efficiency increase with the amount of pre-existing CRs at weak shocks. But the presence of pre-existing CRs does not affect $\xi$ or $P_{c, 2}$ for strong shocks with $M_{s} \gtrsim 10$, in which the freshly injected particles dominate over the re-accelerated ones.

2. The nonlinear stage of MFA via plasma instabilities at collisionless shocks is not fully understood yet. So we adopted a model for MFA via CR streaming instabilities suggested by Caprioli (2012). We argue that the CR current, $j_{\mathrm{cr}} \sim e \xi \sigma_{2} n_{\mathrm{H}, 0} u_{s}$, is high enough to overcome the magnetic field tension, so the Bell-type instability can amplify turbulent magnetic fields at cosmological shocks considered here (Zweibel \& Everett 2010). For shocks with $M \gtrsim 5$, DSA is efficient enough to develop a significant shock precursor due to the CR feedback, and the amplified magnetic field strength in the upstream region scales as $B_{1} / B_{0} \approx 0.12 M_{A, 0} \approx\left(\beta_{P} / 100\right)^{1 / 2} M_{s}$. This MFA model predicts that the postshock magnetic field strength becomes $B_{2} \approx 2-3 \mu \mathrm{G}$ for the shock models considered here (see Table 1).

3. This study demonstrates that if scattering centers drift with the effective Alfvén speed in the local, amplified magnetic field, the CR energy spectrum can be steepened and the acceleration efficiency is reduced significantly, compared to the cases without MFA. As a result, the CR acceleration efficiency saturates at $\eta=2 e_{c, 2} u_{2} / \rho_{0} u_{s}^{3} \approx 0.2$ for $M_{s} \gtrsim 10$, which is significantly lower than what we reported in our previous study, $\eta \approx 0.55$ (Kang et al. 2007). We note that the value $\eta$ at the strong shock limit can vary by $\sim 10 \%$, depending on the model parameters such as the injection parameter, plasma beta, and wave drift speed. Inclusion of wave dissipation (not considered here) will also affect the extent of MFA and the acceleration efficiency. This tells us that detailed understandings of plasma physical processes are crucial to the study of DSA at astrophysical collisionless shocks.

4. At weak shocks in the test-particle regime $\left(M_{s} \lesssim 3\right)$, the CR pressure is not dynamically important enough to generate significant MHD waves, so the magnetic field is not amplified and the Alfvénic drift effects are irrelevant.

5. Finally, we note that the CR injection and the CR streaming instabilities are found to be less efficient at quasiperpendicular shocks (e.g., Garaté \& Spitkovsky 2012). It is recognized, however, that streaming of CRs is facilitated through locally parallel inclination of turbulent magnetic fields at the shock surface, so the CR injection can be effective even at quasi-perpendicular shocks in the presence of pre-existing large-scale MHD turbulence (Giacalone 2005; Zank et al. 2006). At oblique shocks the acceleration rate is faster and the diffusion coefficient is smaller due to drift motion of particles along the shock surface (Jokipii 1987). In fact, the diffusion convection Equation (1) should be valid for quasi-perpendicular shocks as long as there exists strong MHD turbulence sufficient enough to keep the pitch-angle distribution of particles isotropic. In that case, the time-asymptotic states of the $\mathrm{CR}$ shocks should remain the same even for much smaller $\kappa(x, p)$, as mentioned in Section 2.5. In addition, the perpendicular current-driven instability is found to be effective at quasi-perpendicular shocks (Riquelme \& Spitkovsky 2010; Schure et al. 2012). Thus, we expect that the overall conclusions drawn from this study should be applicable to all non-relativistic shocks, regardless of the magnetic field inclination angle, although our quantitative estimates for the CR injection and acceleration efficiencies may not be generalized to oblique shocks with certainty. 
H.K. was supported by the Basic Science Research Program through the National Research Foundation of Korea (NRF) funded by the Ministry of Education, Science and Technology (2012-001065). D.R. was supported by the National Research Foundation of Korea through grant 2007-0093860. The authors thank D. Capriloi, T. W. Jones, F. Vazza, and the anonymous referee for constructive suggestions and comments. H.K. also thanks Vahe Petrosian and KIPAC for their hospitality during the sabbatical leave at Stanford University, where a part of the paper was written.

\section{REFERENCES}

Ackermann, M., Ajello, M., Allafort, A., et al. 2010, ApJL, 717, L71

Arlen, T., Aune, T., Beilicke, M., et al. 2012, ApJ, 757, 123

Bell, A. R. 1978, MNRAS, 182, 147

Bell, A. R. 2004, MNRAS, 353, 550

Beresnyak, A., Jones, T. W., \& Lazarian, A. 2009, ApJ, 707, 1541

Berezhko, E. G., Ksenofontov, L. T., \& Völk, H. J. 2009, A\&A, 505, 169

Blandford, R. D., \& Eichler, D. 1987, PhR, 154, 1

Brüggen, M., Bykov, A., Ryu, D., \& Röttgering, H. 2012, SSRv, 166, 187

Brunetti, G., Blasi, P., Reimer, O., et al. 2012, MNRAS, 426, 956

Brunetti, G., \& Lazarian, A. 2007, MNRAS, 378, 245

Bykov, A. M., Osipov, S. M., \& Ellison, D. C. 2011, MNRAS, 410, 39

Caprioli, D. 2012, JCAP, 07, 038

Caprioli, D., Blasi, P., Amato, E., \& Vietri, M. 2009, MNRAS, 395, 895

Carilli, C. L., \& Taylor, G. B. 2002, ARA\&A, 40, 319

Cassano, R., \& Brunetti, G. 2005, MNRAS, 357, 1313

Donnert, J., Dolag, K., Cassano, R., \& Brunetti, G. 2010, MNRAS, 407, 1565

Drury, L. O'C. 1983, RPPh, 46, 973

Drury, L. O'C., \& Downes, T. P. 2012, MNRAS, 427, 2308

Feretti, L., Giovannini, G., Govoni, F., \& Murgia, M. 2012, A\&ARv, 20, 54

Gargaté, L., \& Spitkovsky, A. 2012, ApJ, 744, 67

Giacalone, J. 2005, ApJL, 628, L37

Govoni, F., \& Feretti, L. 2004, IJMPD, 13, 1549

Guo, F., Jokipii, J. R., \& Kota, J. 2010, ApJ, 725, 128

Hoeft, M., Brüggen, M., Yepes, G., Gottlober, S., \& Schwope, A. 2008, MNRAS, 391,1511

Jeltema, T. E., \& Profumo, S. 2011, ApJ, 728, 53

Jokipii, J. R. 1987, ApJ, 313, 6842

Jones, T. W. 1993, ApJ, 413, 619
Kang, H. 2012, JKAS, 45, 127

Kang, H., \& Jones, T. W. 2007, APh, 28, 232

Kang, H., Jones, T. W., \& Gieseler, U. D. J. 2002, ApJ, 579, 337

Kang, H., \& Ryu, D. 2011, ApJ, 734, 18

Kang, H., Ryu, D., Cen, R., \& Ostriker, J. P. 2007, ApJ, 669, 729

Kang, H., Ryu, D., Cen, R., \& Song, D. 2005, ApJ, 620, 21

Kang, H., Ryu, D., \& Jones, T. W. 1996, ApJ, 456, 422

Kang, H., Ryu, D., \& Jones, T. W. 2009, ApJ, 695, 1273

Kang, H., Ryu, D., \& Jones, T. W. 2012, ApJ, 756, 97

Kronberg, P. P., Dufton, Q. W., Li, H., \& Colgate, S. A. 2001, ApJ, 560, 178

Lee, S., Ellison, D. C., \& Nagataki, S. 2012, ApJ, 750, 156

Lucek, S. G., \& Bell, A. R. 2000, MNRAS, 314, 65

Malkov, M. A., \& Drury, L. O’C. 2001, RPPh, 64, 429

Markevitch, M., \& Vikhlinin, A. 2007, PhR, 443, 1

Miniati, F., Ryu, D., Kang, H., et al. 2000, ApJ, 542, 608

Ohira, Y., Reville, B., Kirk, J. G., \& Takahara, F. 2009, ApJ, 698, 445

Parizot, E., Marcowith, A., Ballet, J., \& Gallant, Y. A. 2006, A\&A, 453, 387

Petrosian, V. 2001, ApJ, 557, 560

Pfrommer, C., Springel, V., Enßlin, T. A., \& Jubelgas, M. 2006, MNRAS, 367,113

Ptuskin, V. S., \& Zirakashvili, V. N. 2005, A\&A, 429, 755

Ptuskin, V. S., Zirakashvili, V. N., \& Seo, E. 2010, ApJ, 718, 31

Reynolds, S. P., Gaensler, B. M., \& Bocchino, F. 2012, SSRv, 166, 231

Riquelme, M. A., \& Spitkovsky, A. 2009, ApJ, 694, 626

Riquelme, M. A., \& Spitkovsky, A. 2010, ApJ, 717, 1054

Riquelme, M. A., \& Spitkovsky, A. 2011, ApJ, 733, 63

Rogachevskii, I., Kleeorin, N., Brandenburg, A., \& Eichler, D. 2012, ApJ, 753, 6

Ryu, D., Kang, H., Cho, J., \& Das, S. 2008, Sci, 320, 909

Ryu, D., Kang, H., Hallman, E., \& Jones, T. W. 2003, ApJ, 593, 599

Schure, K. M., Bell, A. R., Drury, L. O'C., \& Bykov, A. M. 2012, SSRv, 173,491

Skilling, J. 1975, MNRAS, 172, 557

Skillman, S. W., O’Shea, B. W., Hallman, E. J., Burns, J. O., \& Norman, M. L. 2008, ApJ, 689, 1063

van Weeren, R., Röttgering, H. J. A., Brüggen, M., \& Hoeft, M. 2010, Sci, 330,347

Vazza, F., Brüggen, M., Gheller, C., \& Brunetti, G. 2012, MNRAS, 421, 3375

Vazza, F., Brunetti, G., \& Gheller, C. 2009, MNRAS, 395, 1333

Vladimirov, A. E., Bykov, A. M., \& Ellison, D. C. 2008, ApJ, 688, 1084

Völk, H. J., \& Atoyan, A. M. 1999, APh, 11, 73

Zank, G. P., Li, G., Florinski, V., et al. 2006, JGR, 111, 06108

Zweibel, E. G., \& Everett, J. E. 2010, ApJ, 709, 1412 